in a study of 100 neuromuscular patients with elevated VGCC antibodies, only 6 patients were diagnosed with LEMS. This case illustrates the importance of applying appropriate clinical judgement with results of investigations.

\section{ISCHAEMIC STROKE AS THE ONLY MANIFESTATION OF ANTI-NEUTROPHILIC CYTOPLASMIC AUTOANTIBODY ASSOCIATED VASCULITIS}

${ }^{1}$ John Tran, ${ }^{2}$ Joshua Abasszade, ${ }^{1}$ Yew Li (Michelle) Dang, 'Doug Crompton. 'Northern Health, Melbourne, VIC, Australia; ${ }^{2}$ Monash Health, Melbourne, VIC, Australia

\subsection{6/bmjno-2021-ANZAN.51}

Background The diagnosis of anti-neutrophilic cytoplasmic autoantibodies (ANCA) associated vasculitis (AAV) in first episode strokes is more challenging compared with consecutive strokes, especially in patients lacking other clinical features of AAV.

Methods/Results Here, we present the case of a 71-year-old female with positive myeloperoxidase (MPO) ANCA and negative proteinase 3 (PR3) ANCA. Our patient presented with a one-week history of pyramidal weakness in both upper and lower limbs, hyperreflexia and clonus. Brain MRI demonstrated widespread bi-hemispheric cortical and deep white matter acute infarcts. Investigations revealed eosinophilia on full blood examination and positive MPO-ANCA antibody. Consistent with features of stroke secondary to AAV, the deep penetrating vessels were predominantly affected resulting in a multifocal distribution of infarcts in the white matter. MPOANCA positive vasculitis diseases are more commonly associated with renal, pulmonary and cutaneous manifestations, however our patient did not have other systemic manifestations of AAV, and her presentation was solely limited to the CNS.

Conclusions This case highlights the challenges of diagnosing primary CNS vasculitis, especially an atypical MPO-ANCA positive disease that fails to have the classical clinical signs and course.

\section{PREDICTIVE VALUE OF SIGNS AND SYMPTOMS IN CODE STROKES FOR DIAGNOSIS OF ISCHAEMIC STROKE OR TIA}

${ }^{1}$ Mei Yan Ngun, 'Mark Tacey, ${ }^{2}$ Douglas Crompton. 'Austin Hospital, Heidelberg, VIC, Australia; ${ }^{2}$ Northern Health, Epping, VIC, Australia

\subsection{6/bmino-2021-ANZAN.52}

Objectives This study aimed to determine the history features, signs and symptoms identified during a code stroke that correlate with the diagnosis of ischemic stroke or transient ischemic attack (TIA). We investigated the rate of stroke mimics and aimed to develop a clinical prediction model.

Methods Consecutive code stroke presentations to a primary stroke centre were recruited. Patient characteristics, medical history, signs or symptoms on activation of code stroke were collected from the medical record. Diagnosis of ischemic stroke was determined by radiographic evidence of infarction. Univariate analysis and multivariable logistic regression analysis were used to determine the features that predict ischemic stroke/TIA versus mimic.
Results Among 493 code strokes, 64.5\% were mimics. The most commonly diagnosed mimics were migraine, peripheral vertigo and seizure. Upper limb sensory change (OR 3.27 [95\% CI, 1.75-6.11]), hemiplegia (OR 2.70 [95\% CI, 1.654.43]), dysphasia (OR 2.62 [95\% CI, 1.56-4.40]) and history of atrial fibrillation (OR 2.01 [95\% CI, 1.14-3.54]) or hypertension (OR 1.77 [95\% CI, 1.10-2.83]) are highly predictive of stroke/TIA. Headache (OR 0.40 [95\% CI, 0.23-0.69]) is predictive of a mimic. Dizziness and vertigo were more common in stroke mimics. C-statistic for the study models ranged from 0.70 to 0.76 .

Conclusion Objective signs such as unilateral motor weakness and dysphasia are highly predictive of ischemic stroke/TIA whereas symptoms of headache and dizziness are suggestive of stroke mimic. Stroke mimic rate is influenced by local prevalence and threshold for code stroke activation. Incorporating positive and negative predictive features may improve future stroke prediction tools.

\section{EOSINOPHILIC GRANULOMATOSIS WITH POLYANGIITIS PRESENTING WITH SIMULTANEOUS CENTRAL AND PERIPHERAL NERVOUS SYSTEM INVOLVEMENT}

Julia Lim, Yi Chao Foong, Abhishek Malhotra. Barwon Health, Geelong, VIC, Australia

\subsection{6/bmjno-2021-ANZAN.53}

Objective Peripheral nervous system involvement in eosinophilic granulomatosis with polyangiitis (EGPA) is well described. However, central nervous system involvement is uncommon. We describe a case of simultaneous central and peripheral nervous system involvement in EGPA. The diagnosis was confirmed on histopathology. A high index of suspicion is needed to initiate prompt treatment for this potentially lifethreatening condition.

Report 85-year-old male presented with subacute generalised lower limb weakness on the background of known eosinophilic asthma treated with interleukin-5 inhibitor (mepolizumab) and prednisolone. During his admission, he developed transient aphasia, followed by mononeuritis multiplex involving the left median and femoral nerves and purpuric rash over few days. Laboratory investigations showed no peripheral eosinophilia. ANCA revealed a borderline elevated myeloperoxidase antigen (MPO) of $21 \mathrm{U} / \mathrm{mL}$. MRI brain revealed multiple small foci of diffusion restriction within the basal ganglia bilaterally, as well as paranasal sinusitis. MRA/CT cerebral angiogram was unremarkable. Prolonged telemetry and TOE did not show any central embolic cause. Left lateral gastrocnemius muscle biopsy revealed fibrinoid necrosis associated with adjacent eosinophils. Induction with intravenous cyclophosphamide was commenced along with high dose corticosteroids. $\mathrm{He}$ has been neurologically stable since.

Conclusion Simultaneous peripheral nervous system involvement with multiterritory stroke should heighten the suspicion for systemic vasculitis. Cerebral arterial imaging may be normal in small to medium vessel vasculitis such as EGPA. Preexisting mepolizumab therapy may make diagnosis more challenging by normalising pathology results. Histopathology can be of value to confirm diagnosis.

\section{REFERENCES}

1. Keogh KA, Specks U. Churg-Strauss syndrome: update on clinical, laboratory and therapeutic aspects. Sarcoidosis Vasc Diffuse Lung Dis 2006 Mar;23(1):3-12. 
2. André R, Cottin V, Saraux JL, et al. Central nervous system involvement in eosinophilic granulomatosis with polyangiitis (Churg-Strauss): Report of 26 patients and review of the literature. Autoimmun Rev 2017;16(9):963-969.

\section{AN ADULT CASE OF ACUTE CEREBELLITIS AS A MANIFESTATION OF MYCOPLASMA PNEUMONIAE INFECTION}

Amanda Ji, Michael Roizman, Andrew Swayne. Centre for Neurosciences, Mater Hospital, Brisbane, QLD, Australia

\subsection{6/bmjno-2021-ANZAN.54}

Introduction Acute cerebellitis is exceptionally rare in adults often preceded by systemic viral or bacterial infections. We present a case of acute cerebellitis following otherwise asymptomatic Mycoplasma Pneumoniae infection.

Case Descriptions A 40-year-old man presented with a threeday history of acute onset of dizziness, vomiting and headache which was initially diagnosed and managed as vestibular neuritis. He was discharged and four days later represented with slurred speech and difficulty walking. On examination, he was alert, had an ataxic gait, dysarthria and bilateral hypermetric saccades. CSF studies showed an elevated leukocyte count of $152 \times 10^{6} / \mathrm{L}$ with $100 \%$ mononuclear cells and a mildly elevated protein level of $0.7 \mathrm{~g} / \mathrm{L}$ with normal glucose. Other CSF analysis including a multiplex PCR panel for a variety of viral, bacterial and fungal pathogens, cytology, flow cytometry, antineuronal and encephalitis antibody tests were negative. MRI showed features consistent with acute cerebellitis including subtle diffuse cerebellar hyperintense signal on T2 and FLAIR sequences. Serological testing showed the presence of Mycoplasma Pneumoniae IgM and IgG. A diagnosis of acute cerebellitis was made, and the patient received supportive care and made a complete neurological recovery after 10 days. A repeat MRI one month later showed imaging resolution and he remained asymptomatic at clinic follow-up.

Conclusion This case describes an adult with cerebellitis most likely secondary to Mycoplasma Pneumoniae infection with other causes excluded on extensive investigation. Although rare, acute cerebellitis should be considered as a part of the differential diagnosis of acute vertigo particularly in the presence of cerebellar signs.

\section{AN ATYPICAL PRESENTATION OF TRANSIENT EPILEPTIC AMNESIA LASTING SIX HOURS}

Benjamin D Wood, Amanda Ji, Michael Roizman, Andrew Swayne. Mater Centre for Neurosciences, Brisbane, QLD, Australia

\subsection{6/bmjno-2021-ANZAN.55}

Background Transient epileptic amnesia (TEA) is a clinical presentation of focal epilepsy from temporal origin characterised by transient episodes of anterograde amnesia without other aspects of cognitive impairment. It is a rare diagnosis which requires evidence of epilepsy, such as epileptiform abnormalities on electroencephalogram (EEG). We present a case of a 76-year-old female with a prolonged episode of TEA.

Case Description A previously well 76-year-old female presented to a tertiary hospital emergency department following sudden onset anterograde amnesia. She was disoriented to time and had repetitive questioning, however routine physical and neurological examination revealed no abnormalities. Initial investigations including CT Head and routine bloods did not find a reversible cause for her symptoms. She subsequently fully recovered after six hours. MRI of her brain showed chronic small vessel changes with no hippocampal abnormality. Routine EEG revealed right temporal sharp waves maximal at T8, indicating an underlying temporal lobe epilepsy. A diagnosis of TEA was made, and the patient was commenced on Levetiracetam. The patient has not had further amnestic events since.

Conclusion This case demonstrates an atypical presentation of TEA. Typically, episodes of TEA last less than $1 \mathrm{hr}$ and occur on waking. Our case presented deceptively similarly to Transient Global Amnesia, a disorder with usually one episode of anterograde amnesia 2-24hrs long, characterized by repetitive questioning without impairment of other cognitive functions. However routine EEG workup showed evidence of temporal lobe epilepsy, re-clarifying the diagnosis as TEA. This case demonstrates the importance of EEG workup in presentations of transient amnesia.

\section{OUTCOME OF ENDOVASCULAR THROMBECTOMY FOR ULTRA-LONG AEROMEDICAL TRANSFERS: THE EXPERIENCE OF ONE 'HUB' AND ELEVEN 'SPOKE' SITES COVERING MORE THAN 1.8 MILLION KM ${ }^{2}$}

${ }^{1}$ Seppy Pakrah, ${ }^{1}$ Karen Huang, ${ }^{1}$ Haylee Berrill, 'Meng Tan, ${ }^{1}$ Peter Bailey, ${ }^{1}$ Arman Sabet, ${ }^{2}$ Laetitia Devilliers, ${ }^{2} \mathrm{Hal}$ Rice, ${ }^{1}$ Darshan Shah. ${ }^{1}$ Department of Neurology, Gold Coast University Hospital, Southport, QLD, Australia; ${ }^{2}$ Department of Interventional Neuroradiology, Gold Coast University Hospital, Southport, QLD, Australia

\subsection{6/bmjno-2021-ANZAN.56}

Background Local endovascular services for acute stroke in rural and remote regions remain a significant challenge despite higher rates of stroke in these regions.

Objectives We present time metrics, logistics, safety and outcome data on stroke patients with large vessel occlusion (LVO) that were aero-medically transferred from rural and remote 'spoke' sites to the Gold Coast University Hospital (GCUH) 'hub' for endovascular thrombectomy (EVT).

Methods This is a retrospective observational study utilizing prospectively collected stroke database from December 2018 to March 2020. Aeromedical transfers from ten rural and remote Queensland sites and one Northern New South Wales site were included, covering more than 1.8 million $\mathrm{km}^{2}$ catchment area.

Results Over the period of 16 months, 20 out of 26 transferred patients underwent EVT. Mean distance was $1350 \mathrm{~km}$, median time of ictus to recanalization was 928 minutes and TICI $2 \mathrm{~b}-3$ was achieved in $90 \%$ of the patients. One out of 20 patients $(5 \%)$ had symptomatic intracranial hemorrhage (sICH), and 9 out of $20(45 \%)$ achieved functional independence (mRS 0-2) at 90 days; similar to the recent pivotal trials.

Conclusions Our 12-center network experience confirms real world reproducibility of trial results, including ultra-long transfers, and supports such transfers at other centers worldwide. 